\title{
Hwang-Heuk-San induces apoptosis in HCT116 human colorectal cancer cells through the ROS-mediated activation of caspases and the inactivation of the PI3K/Akt signaling pathway
}

\author{
MOON HEE LEE ${ }^{1}$, SU-HYUN HONG ${ }^{1}$, CHEOL PARK $^{2}$, GI-YOUNG KIM ${ }^{3}$, SUN-HEE LEEM ${ }^{4}$, \\ SUNG HYUN $\mathrm{CHOI}^{5}$, YOUNG-SAM KEUM ${ }^{6}$, JIN WON HYUN ${ }^{7}$, TAEG KYU KWON ${ }^{8}$, \\ SANG HOON $\mathrm{HONG}^{9}$ and YUNG HYUN CHOI ${ }^{1,10}$
}

\author{
${ }^{1}$ Department of Biochemistry, Dongeui University College of Korean Medicine, Busan 614-052; \\ ${ }^{2}$ Department of Molecular Biology, Dongeui University, Busan 614-714; ${ }^{3}$ Department of Marine Life Sciences, \\ Jeju National University, Jeju 690-756; ${ }^{4}$ Department of Biological Science, Dong-A University, Busan 604-714; \\ ${ }^{5}$ Department of Safety and System Management, Korea Lift College, Geochang 670-802; ${ }^{6}$ College of Pharmacy, \\ Dongguk University, Goyang 410-773; ${ }^{7}$ Department of Biochemistry, School of Medicine, Jeju National University, \\ Jeju 609-756; ${ }^{8}$ Department of Immunology, School of Medicine, Keimyung University, Daegu 704-701; \\ ${ }^{9}$ Department of Internal Medicine, Dongeui University College of Korean Medicine, Busan 614-052; \\ ${ }^{10}$ Anti-Aging Research Center \& Blue-Bio Industry RIC, Dongeui University, Busan 614-714, Republic of Korea
}

Received January 8, 2016; Accepted February 22, 2016

DOI: $10.3892 / o r .2016 .4812$

\begin{abstract}
Hwang-Heuk-San (HHS) is a polyherbal formulation that has been used in traditional Korean medicine for hundreds of years to treat gastrointestinal malignancy. However, to date, the mechanisms responsible for the anticancer effects remain unclear.In the present study, we investigated the anticancer effects of HHS using HCT116 human colorectal cancer (CRC) cells. Our results showed that HHS treatment significantly reduced cell survival and increased apoptotic cell death in a concentrationdependent manner. The treatment of HCT116 cells with HHS also significantly elevated the accumulation of reactive oxygen species (ROS), which was followed by the attenuation of the mitochondrial membrane potential through the upregulation of Bax and the downregulation of Bcl-2, which was accompanied by the release of cytochrome $c$ to the cytosol. In addition, HHS treatment caused the truncation of Bid and activated the caspases (caspase-8, -9 and -3 ), which was associated with the induction of the Fas ligand, the death receptors (DRs), DR4 and DR5, downregulation of the inhibitors of protein expression in the apoptosis protein family, and the degradation of poly(ADPribose)-polymerase. However, a pan-caspase inhibitor reversed the HHS-induced apoptosis and growth suppression, indicating that HHS induces apoptosis though a caspase-dependent intrinsic and extrinsic apoptotic pathway in HCT116 cells. Moreover,
\end{abstract}

Correspondence to: Dr Yung Hyun Choi, Department of Biochemistry, Dongeui University College of Korean Medicine, San 45, Yangjung-dong, Busanjin-gu, Busan 614-052, Republic of Korea E-mail: choiyh@deu.ac.kr

Key words: Hwang-Heuk-San, colorectal cancer cells, apoptosis, PI3K/Akt, ROS
HHS treatment inhibited the activation of phosphatidylinositol3-kinase (PI3K)/Akt signaling, and a pharmacological inhibitor of PI3K significantly potentiated the apoptotic effects of HHS when employed in combination in HCT116 cells. Furthermore, the blocking of ROS generation by antioxidant $\mathrm{N}$-acetyl cysteine attenuated the HHS-induced release of cytochrome $c$, caspase activation and PI3K/Akt inactivation, thereby preventing HHS-induced apoptosis and reduction in cell viability. These findings suggest that HHS-induced ROS generation is required for caspase-dependent apoptotic cell death involving inhibition of the PI3K/Akt signaling pathway in HCT116 cells. Overall, our findings suggest that HHS may be an effective treatment for $\mathrm{CRC}$ cancer, and further studies are required to identify the active compounds in HHS.

\section{Introduction}

Colorectal cancer (CRC) is presently the third most frequently diagnosed cancer and the fourth leading cause of cancer-related deaths worldwide (1). The incidence of CRC is now rapidly increasing in previously known low-risk areas, such as Asia, Latin America and Africa (2). Despite considerable progress in clarifying the critical factors involved in the prevention of different types of CRCs, this mortality is primarily attributed to the high incidence of metastasis due to its acquired resistance to anticancer drugs (3). Therefore, the development of novel therapeutic strategies with increased selectivity and reduced toxicity for $\mathrm{CRC}$ treatment is required.

One of the hallmarks of cancer is the evasion of tumor cells by apoptosis, a highly regulated process of programmed cell death. The disruption of this process is a major contributing factor in the pathology of cancer (4). It has been well established that the apoptotic process triggers extrinsic [death 
receptor (DR)-mediated] or intrinsic (mitochondria-dependent) caspase-dependent pathways $(5,6)$. In addition, the integrity of the mitochondrial membrane is regulated by the interactions of Bcl-2 family proteins. Reactive oxygen species (ROS) derived from mitochondrial oxidative metabolism are also an important mediator of apoptosis induction (7). Among several cellular signaling pathways, the phosphatidylinositol-3-kinase (PI3K)/ Akt signal transduction pathway integrates the survival signals provided by extracellular and intracellular stimuli to promote cell growth and to inhibit cell death, which is mediated by intracellular ROS generation $(7,8)$. Moreover, the PI3K/Akt pathway is highly activated in most human cancers, including CRC $(9,10)$. Current research is highly interested in exploiting this pathway for the discovery of potential cancer treatment. Therefore, agents that target this apoptosis pathway without affecting normal cells play crucial roles as potential drug targets in cancer treatment.

For thousands of years, herbal medicines have been used with apparent safety and efficacy to alleviate and treat various diseases, including cancer, in numerous countries $(11,12)$. In particular, typical traditional herbal medicinal prescriptions consist of several components that are combined to minimize side-effects, maximize therapeutic efficacy by facilitating synergistic actions between the drugs while preventing potential adverse effects, and improve the quality of life of patients (13). Among such components, Hwang-Heuk-San (HHS) is an aqueous polyherbal formulation that consists of crude ingredients extracted from four medicinal herbs (Table I), which are described in the Donguibogam, an ancient Korean medical book (14). HHS has been used for hundreds of years in Korea for the clinical treatment of intestinal abscesses and carbuncles on the abdominal wall. However, despite its valuable clinical effects on patients, few attempts have been made to investigate the molecular mechanism and pharmacological action of HHS in gastrointestinal malignancy.

The aim of the present study was to discover novel biologically active substances in traditional medicinal resources for the prevention and treatment of cancers. We aimed to determine whether HHS could inhibit the growth of and trigger apoptosis in HCT-116 CRC cells. The results showed that HHS triggered caspase-dependent apoptosis through the activation of both intrinsic and extrinsic pathways. The present study is the first to investigate the potential roles and underlying mechanisms connecting ROS generation and the PI3K/Akt pathway in mediating HHS-induced apoptosis in HCT-116 cells.

\section{Materials and methods}

Reagents and antibodies. RPMI-1640 medium, fetal bovine serum (FBS), penicillin and streptomycin were purchased from WelGENE Inc. (Daegu, Korea); 3-(4,5-dimethylthiazol-2-yl)-2,5-diphenyltetrazolium bromide (MTT), 4',6-diamidino-2-phenylindole (DAPI), phenol-chloroform, isoamyl alcohol, RNase A, dithiothreitol (DTT), propidium iodide (PI), paraformaldehyde, 5,5',6,6'-tetrachloro1,1',3,3'-tetraethyl-benzimidazolyl carbocyanine iodide (JC-1) and N-acetylcysteine (NAC), a ROS scavenger were purchased from Sigma-Aldrich Chemicals Co.(St.Louis, MO,USA).z-ValAla-Asp-fluoromethylketone (z-VAD-fmk), a pan-caspase inhibitor and LY294002, a selective inhibitor of PI3K, were obtained from Calbiochem (San Diego, CA, USA) and Cell
Table I. Herbal components and amount of HHS decoction.

Herbal medicine

Raw material amount

(pharmacognostic nomenclature) $(\mathrm{g} / \%)$

Rheum palmatum $\mathrm{L}$. $36.0(47.4)$

(Rhei Radix et Rhizoma)

Psoralea corylifolia L.

(Psoraleae Fructus)

Pharbitis nil Chois.

(Pharbitidis Semen)

Arctium lappa L.

$8.0 \quad(10.5)$

(Arctii Fructus)

Total amounts

$76.0(100.0)$

HHS, Hwang-Heuk-San

Signaling Technology, Inc. (Danvers, MA, USA), respectively. 2',7'-Dichlorodihydrofluorescein diacetate (DCF-DA) and the enhanced chemiluminescence (ECL) kit were purchased from Molecular Probes (Leiden, The Netherlands) and Amersham Pharmacia Biotech (Arlington Heights, IL, USA), respectively. Primary antibodies (Table II) were purchased from Santa Cruz Biotechnology, Inc. (Santa Cruz, CA, USA), Cell Signaling Technology, Inc., Bioworld Technology, Inc. (St. Louis Park, MN, USA) and Abcam (Cambridge, UK). Peroxidase-labeled donkey anti-rabbit and sheep anti-mouse immunoglobulin were purchased from Amersham Pharmacia Biotech. All other chemicals not mentioned in the present study were purchased from Sigma-Aldrich Chemical Co.

Preparation of the HHS. The four medicinal herbs forming HHS were obtained from Dongeui Oriental Hospital, Dongeui University College of Korean Medicine (Busan, Korea). The origin of the medicinal herbs was taxonomically confirmed by Professor Su Hyun Hong at the Dongeui University College of Korean Medicine (Busan). Each of the four herbs in HHS was cut into small pieces, which were mixed together to obtain a total amount of $76 \mathrm{~g}$ in the ratios shown in Table I. The mixture was boiled in distilled water $(76 \mathrm{~g} / 500 \mathrm{ml})$ for $3 \mathrm{~h}$. The extract solution was filtered using a $0.45 \mu \mathrm{M}$ filter to remove insoluble materials, and the blended supernatants were lyophilized and and then crushed into a thin powder (yield, $21 \% \mathrm{w} / \mathrm{w}$, dried extract/crude herb). The dried extract (HHS) was dissolved in a $100 \mathrm{mg} / \mathrm{ml}$ concentration with distilled water, and the stock solution was then diluted with medium to the desired concentration prior to use.

Cell culture and cell viability assay. CRC HCT116 cells were purchased from the American Type Culture Collection (ATCC; Manassas, MD, USA) and maintained at $37^{\circ} \mathrm{C}$ in an incubator with a humidified atmosphere of $5 \% \mathrm{CO}_{2}$ in air. The cells were cultured in RPMI-1640 medium containing $10 \%$ FBS, $100 \mathrm{U} / \mathrm{ml}$ of penicillin and $100 \mathrm{mg} / \mathrm{ml}$ of streptomycin in the presence or absence of HHS. Cell viability was determined using the MTT assay, which is based on the reduction of a tetrazolium salt by mitochondrial dehydrogenases in viable cells. In brief, HCT116 cells were seeded into a 96-well plate at a density of $1 \times 10^{5}$ cells/well and treated with HHS or reagents. 
Table II. Antibodies used in the present study.

\begin{tabular}{|c|c|c|c|}
\hline Antibody & Dilution & Product no. & Species of origin and supplier \\
\hline Actin & $1: 50000$ & BS6007M & Mouse polyclonal, Bioworld Technology, Inc. \\
\hline Caspase-3 & 1:1000 & sc-7272 & Mouse polyclonal, Santa Cruz Biotechnology, Inc. \\
\hline Caspase-8 & $1: 1000$ & sc-7890 & Rabbit polyclonal, Santa Cruz Biotechnology, Inc. \\
\hline Caspase-9 & 1:1000 & sc-7885 & Rabbit polyclonal, Santa Cruz Biotechnology, Inc. \\
\hline PARP & $1: 1000$ & sc-7150 & Rabbit polyclonal, Santa Cruz Biotechnology, Inc. \\
\hline XIAP & $1: 1000$ & sc-11426 & Rabbit polyclonal, Santa Cruz Biotechnology, Inc. \\
\hline cIAP-1 & 1:1000 & sc-7943 & Rabbit polyclonal, Santa Cruz Biotechnology, Inc. \\
\hline cIAP-2 & $1: 1000$ & sc-7944 & Rabbit polyclonal, Santa Cruz Biotechnology, Inc. \\
\hline survivin & 1:1000 & sc-17779 & Mouse polyclonal, Santa Cruz Biotechnology, Inc. \\
\hline TRAIL & 1:1000 & sc-7877 & Rabbit polyclonal, Santa Cruz Biotechnology, Inc. \\
\hline DR4 & $1: 1000$ & sc-7863 & Rabbit polyclonal, Santa Cruz Biotechnology, Inc. \\
\hline DR5 & 1:1000 & sc-65314 & Mouse polyclonal, Santa Cruz Biotechnology, Inc. \\
\hline Fas & $1: 1000$ & sc-715 & Rabbit polyclonal, Santa Cruz Biotechnology, Inc. \\
\hline FasL & $1: 1000$ & sc-957 & Rabbit polyclonal, Santa Cruz Biotechnology, Inc. \\
\hline Bcl-2 & $1: 1000$ & sc-783 & Rabbit polyclonal, Santa Cruz Biotechnology, Inc. \\
\hline Bax & 1:1000 & sc-493 & Rabbit polyclonal, Santa Cruz Biotechnology, Inc. \\
\hline Bid & $1: 1000$ & sc-11423 & Rabbit polyclonal, Santa Cruz Biotechnology, Inc. \\
\hline Cytochrome c & $1: 1000$ & sc-7159 & Rabbit polyclonal, Santa Cruz Biotechnology, Inc. \\
\hline COX IV & $1: 500$ & $20 \mathrm{E} 8 \mathrm{C} 12$ & Mouse monoclonal, Abcam \\
\hline PI3K & $1: 1000$ & $4257 \mathrm{P}$ & Rabbit polyclonal, Cell Signaling Technology, Inc. \\
\hline $\mathrm{p}-\mathrm{PI} 3 \mathrm{~K}$ & 1:1000 & $4228 \mathrm{P}$ & Rabbit polyclonal, Cell Signaling Technology, Inc. \\
\hline Akt & 1:1000 & sc-8312 & Rabbit polyclonal, Santa Cruz Biotechnology, Inc. \\
\hline p-Akt & $1: 1000$ & sc-101629 & Rabbit polyclonal, Santa Cruz Biotechnology, Inc. \\
\hline
\end{tabular}

After incubating for $48 \mathrm{~h}$, the medium was discarded, and the cells were washed with phosphate-buffered saline (PBS), followed by incubation with MTT $(0.5 \mathrm{mg} / \mathrm{ml})$ at $37^{\circ} \mathrm{C}$. After incubating for a further $3 \mathrm{~h}$, the supernatants were aspirated, and the formazan crystals in each well were dissolved in dimethyl sulfoxide (DMSO). Absorbance at $540 \mathrm{~nm}$ was read on an enzyme-linked immunosorbent assay (ELISA) reader (Molecular Devices, Sunnyvale, CA, USA).

Nuclear morphology analysis of apoptosis. For the assessment of apoptosis, the cells were washed with PBS, and the cell suspension was cytospun onto clean fat-free glass slides using a cytocentrifuge. The cells were fixed with $3.7 \%$ paraformaldehyde in PBS and then stained with $2.5 \mu \mathrm{g} / \mathrm{ml}$ of DAPI solution for $10 \mathrm{~min}$ at room temperature. The cells were washed twice with PBS, and the images were then captured using a fluorescence microscope (Carl Zeiss, Jena, Germany).

DNA flow cytometric detection of apoptosis. To analyze apoptotic sub-G1 hypodiploid cells, the cells were harvested, washed twice with ice-cold PBS, and fixed with $75 \%$ ethanol at $4^{\circ} \mathrm{C}$ for $30 \mathrm{~min}$. The cells were then incubated with $1 \mathrm{U} / \mathrm{ml}$ of RNase A (DNase-free) and $10 \mu \mathrm{g} / \mathrm{ml}$ of PI overnight at room temperature in the dark. A flow cytometric analysis was performed using a flow cytometer (FACSCalibur; Becton-Dickinson, San Jose, CA, USA). The sub-G1 hypodiploid cells were assessed based on histograms generated by the CellQuest and ModFit computer programs (Becton-Dickinson). The level of apoptotic cells containing sub-G1 DNA content was determined as a percentage of the total number of cells. The cells were also stained with $5 \mu \mathrm{l}$ of Annexin V-fluorescein isothiocyanate (FITC) (R\&D Systems, Minneapolis, MN, USA) and $5 \mu \mathrm{l}$ of
PI. Following incubation for $15 \mathrm{~min}$ at room temperature in the dark, the degree of apoptosis was quantified as a percentage of the Annexin V-positive and PI-negative (Annexin $\mathrm{V}^{+} / \mathrm{PI}^{-}$cells) cells using a flow cytometer (15).

Protein extraction and western blot analysis. The cells were harvested and lysed with lysis buffer [40 $\mathrm{mM}$ Tris (pH 8.0), $120 \mathrm{mM} \mathrm{NaCl}, 0.5 \%$ Nonidet P-40, $0.1 \mathrm{mM}$ sodium orthovanadate, $2 \mu \mathrm{g} / \mathrm{ml}$ aprotinin, $2 \mu \mathrm{g} / \mathrm{ml}$ leupeptin and $100 \mu \mathrm{g} /$ $\mathrm{ml}$ phenymethylsulfonyl fluoride] for $30 \mathrm{~min}$. In a parallel experiment, the mitochondrial and cytosolic fractions were isolated using a mitochondrial fractionation kit (Active Motif, Carlsbad,CA,USA) according to the manufacturer's instructions. The protein concentration was measured using a Bio-Rad protein assay (Bio-Rad, Hercules, CA, USA). For the western blot analysis, equal amounts of protein (30-50 $\mu \mathrm{g} /$ lane) were fractionated in sodium dodecyl sulphate (SDS)-polyacrylamide gels prior to transfer to the nitrocellulose membranes (Schleicher \& Schuell, Keene, NH, USA). The membranes were blocked with Tris-buffered saline (10 mM of Tris-Cl, pH 7.4) containing $0.5 \%$ Tween-20 and 5\% non-fat dry milk for $1 \mathrm{~h}$ at room temperature and then probed with the desired antibodies for $1 \mathrm{~h}$. The membranes were then washed with PBS and incubated with the secondary antibody conjugated to horseradish peroxidase for $1 \mathrm{~h}$ at room temperature. Immunoreactivity was detected using the ECL western blotting detection system according to the manufacturer's instructions.

Measurement of mitochondrial membrane potential (MMP, $\Delta \psi m)$. The MMP values were determined using the lipophilic cationic probe JC-1, a radiometric dual-emission fluorescent dye that is absorbed and concentrated by respiring mitochon- 


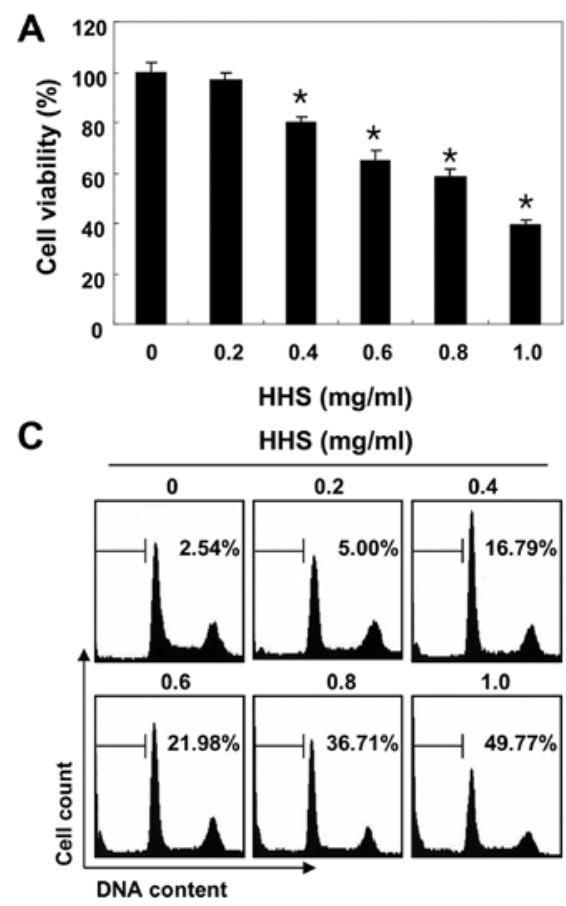

B

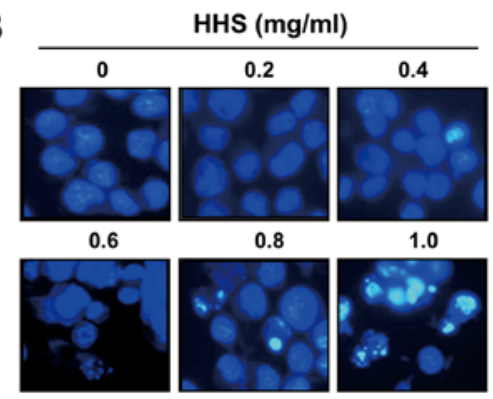

D

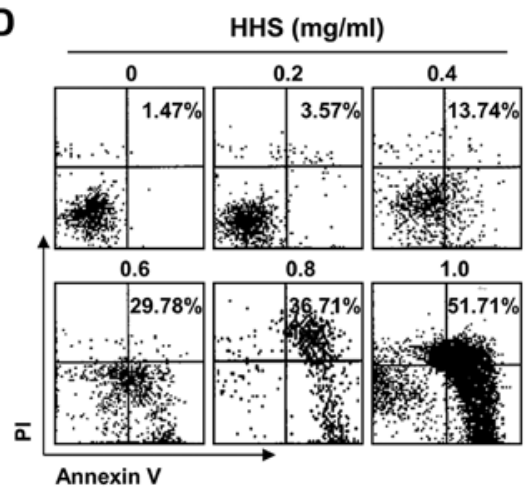

Figure 1. Inhibition of cell viability and induction of apoptosis by HHS treatment in HCT116 cells. (A) The cells were treated with various concentrations of HHS for 48 h. Cell viability was determined by the MTT assay. Statistical significance was determined using one-way ANOVA ( ${ }^{*}<<0.05$ vs. untreated control). (B) The cells grown under the same conditions as (A) were fixed and stained with DAPI to visualize DNA. The stained nuclei were then observed under a fluorescence microscope using a blue filter (original magnification, $\mathrm{x} 400$ ). (C) To quantify the degree of apoptosis induced by HHS, the cells were evaluated by a flow cytometer to determine sub-G1 DNA content, which represents the cells undergoing apoptotic DNA degradation. (D) The cells were also stained with Annexin V-FITC and PI, and the percentages of apoptotic cells (Annexin $\mathrm{V}^{+}$cells) were then analyzed using flow cytometric analysis. (C and D) The results are expressed as the mean of two different experiments.

dria and can reflect MMP changes in cells. Briefly, the cells were fixed and permeabilized with $0.2 \%$ Triton X-100 in PBS for $10 \mathrm{~min}$ at room temperature and then incubated with $10 \mu \mathrm{M}$ of JC-1 for $30 \mathrm{~min}$ at $37^{\circ} \mathrm{C}$ in the dark. Subsequently, the cells were washed with PBS to remove unbound dye, and the amount of JC-1 retained by 10,000 cells/sample was measured at 488 and $575 \mathrm{~nm}$ using a flow cytometer (16).

Determination of caspase activity. The enzymatic activity of the caspases induced by HHS was assayed using colorimetric assay kits (R\&D Systems). Briefly, the cells were lysed in the supplied lysis buffer according to the manufacturer's instructions. The supernatants were collected, and equal amounts of protein were incubated with the supplied reaction buffer containing colorimetric tetrapeptides, Asp-Glu-Val-Asp (DEAD)-p-nitroaniline (pNA) for caspase-3, Ile-Glu-Thr-Asp (IETD)-pNA for caspase-8 and Leu-Glu-His-Asp (LEHD)-pNA for caspase-9. The caspase activity was determined by measuring changes in absorbance at $405 \mathrm{~nm}$ using the ELISA reader.

Measurement of intracellular ROS. The fluorescent probe DCF-DA method was used to measure the level of intracellular ROS in the cells as previously described (17). Twenty-four hours after plating, the cells were treated with $1 \mathrm{mM}$ NAC for $1 \mathrm{~h}$; HHS was then added, and the cells were incubated for another $48 \mathrm{~h}$. After the media were changed, $10 \mu \mathrm{M}$ DCF-DA was added to each well, and the cells were incubated for an additional $30 \mathrm{~min}$ at $37^{\circ} \mathrm{C}$. After washing with PBS, the cells were trypsinized and resuspended in PBS. The fluorescence was measured at specific time intervals using a flow cytometer.
Statistical analysis. The data derived from at least three independent experiments are expressed as mean \pm standard deviation (SD). Statistical comparisons between different groups were performed using a one-way ANOVA, which was followed by Student's t-tests after each treated group was compared to the negative control. Values of $p<0.05$ were considered to indicate a statistically significant result.

\section{Results}

HHS suppresses cell viability and induces apoptosis of the HCT116 cells. To evaluate the effect of HHS on cell viability, the cells were treated with various concentrations of HHS for $48 \mathrm{~h}$, and an MTT assay was performed. As shown in Fig. 1A, the results indicated that HHS exhibited cytotoxic effects in a concentration-dependent manner. In addition, DAPI staining revealed that the apparent morphological changes, such as nuclear condensation and fragmentation, were concentration-dependently increased in the cells treated with HHS, indicating an increasing number of apoptotic cells, whereas these features were not observed in the control cells (Fig. 1B). To measure apoptotic cell death upon HHS treatment, we stained the cells with PI, and the cell cycle profiles were analyzed using a flow cytometer. The results showed that HHS increased the percentage of cells in the apoptotic hypodiploid sub-G1 phase in a dose-dependent manner (Fig. 1C). Annexin V-FITC/PI double staining was also performed to assess the rate of apoptotic cell death, and a significant degree of apoptosis was detected in the HHS-treated cells (Fig. 1D). 
A

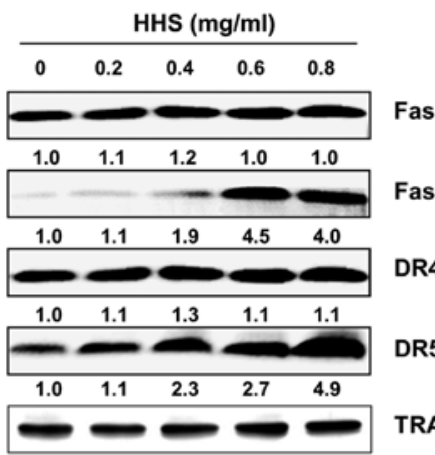

C

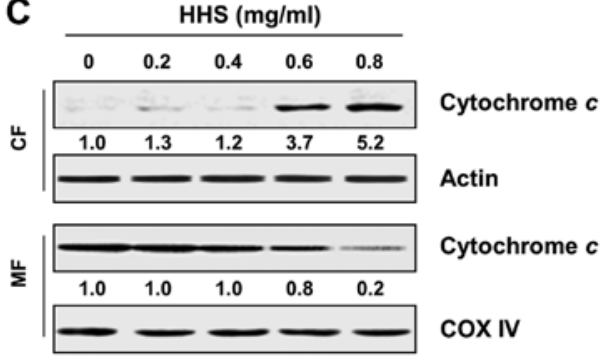

B
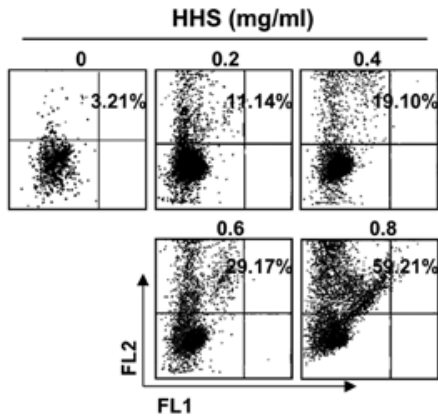

D

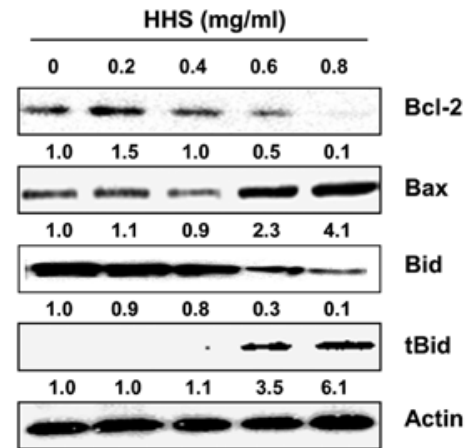

Figure 2. Effects of HHS treatment on the levels of DR-related and Bcl-2 family proteins, MMP and cytochrome $c$ in the HCT116 cells. After incubation with the indicated concentrations of HHS for $48 \mathrm{~h}$, the total cellular proteins (A and D) and cytosolic and mitochondrial proteins (C) were extracted and separated by SDS-polyacrylamide gel electrophoresis, followed by western blot analysis using the indicated antibodies and an ECL detection system. Actin and cytochrome $c$ oxidase subunit 4 (COX IV) were used as internal controls for the cytosolic and mitochondrial fractions, respectively. The numbers represent the average densitometric analyses as compared with actin or COX IV in, at a minimum, two or three different experiments. (B) The cells were collected and incubated with $10 \mu \mathrm{M} \mathrm{JC}-1$ for $20 \mathrm{~min}$ at $37^{\circ} \mathrm{C}$ in the dark. The cells were then washed with PBS, and the mean JC-1 fluorescence intensity was detected using a flow cytometry. The results are represented as the means of two independent experiments.

HHS induces DR-mediated apoptosis in the HCT116 cells. To elucidate the mechanism of HHS-induced apoptosis in the HCT116 cells, we determined whether HHS affects the expression of DR-related proteins. As shown in Fig. 2A, the treatment of HCT116 cells with HHS increased the expression of the Fas ligand (FasL), DR4 and DR5 in a concentration-dependent manner, whereas the levels of Fas and tumor necrosis factorrelated apoptosis-inducing ligand (TRAIL) were relatively unchanged in response to the HHS treatment. The western blot analysis also revealed that the exposure of HCT116 cells to HHS caused the progressive accumulation of truncated Bid (tBid), a BH3 interacting domain death agonist, presumably resulting from truncation by the activated caspase- 8 , in the HHS-treated cells (Fig. 2D).

HHS induces mitochondria-mediated apoptosis in HCT116 cells. To confirm whether HHS induced intrinsic mitochondrial-mediated apoptosis, we determined the effect of HHS on MMP, a hallmark of intrinsic apoptosis, by staining with JC-1 dye. The flow cytometric results demonstrated that HHS induced a significant reduction in the MMP levels with increased concentrations of HHS (Fig. 2B), suggesting the depolarization of the mitochondria by HHS. Since the loss of MMP promotes the release of pro-apoptotic proteins, such as cytochrome $c$ into the cytosol $(18,19)$, we determined the levels of cytochrome $c$ in the cytosolic and mitochondrial fractions of HCT116 cells that were treated with different concentrations of HHS. As shown in Fig. 2C, HHS promoted a concentrationdependent increase in the release of cytochrome $c$ from the mitochondria into the cytosol. Since the $\mathrm{Bcl}-2$ family proteins play a crucial role in mitochondrial homeostasis $(20,21)$, the effect of HHS on the levels of Bcl-2 family proteins was also monitored. Our results showed that the incubation of the HCT116 cells with HHS caused a marked reduction in the level of the anti-apoptotic Bcl-2 protein and increased the level of the pro-apoptotic Bax protein (Fig. 2D).

HHS-induced apoptosis is caspase-dependent in the HCT116 cells. We then examined the expression and activity of caspases in the HHS-treated HCT116 cells to determine whether HHS-induced apoptosis was associated with the activation of the caspases. The results showed that HHS treatment downregulated the levels of pro-caspase- 8 and -9 , which are the initiator caspases of the extrinsic and intrinsic pathway, respectively, in a concentration-dependent manner (Fig. 3A). The subsequent immunoblotting analysis showed that the levels of pro-caspase-3, an effector caspase, were also downregulated in the HHS-treated cells. Correspondingly, the HHS treatment led to the progressive proteolytic cleavage of poly-(ADP-ribose) polymerase (PARP, 113-kDa), a known caspase-3 substrate (22), which yielded an $85-\mathrm{kDa}$ cleaved fragment. For the further quantification of the proteolytic activation of caspases, the lysates equalized by the protein from the cells treated without or with HHS were assayed for their enzymatic activities. As shown in Fig. 3B, the HHS treatment resulted in the significantly increased activity of caspase-8, -9 and -3 . In addition, we examined the expression of inhibitor of the apoptosis protein (IAP) family proteins, which bind to caspases and lead to their inactivation $(23,24)$. As shown in Fig. 3C, HHS treatment reduced the expression of the IAP family proteins, such as XIAP, cIAP-1, cIAP-2 and survivin, 
A

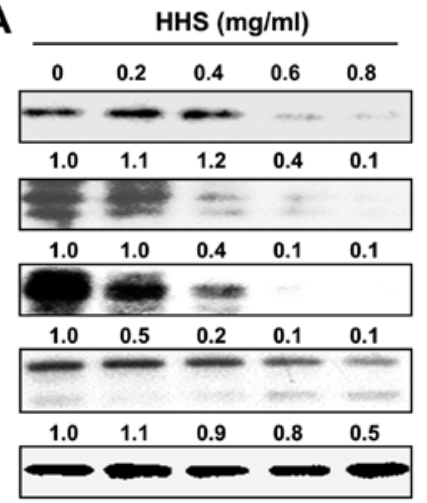

B

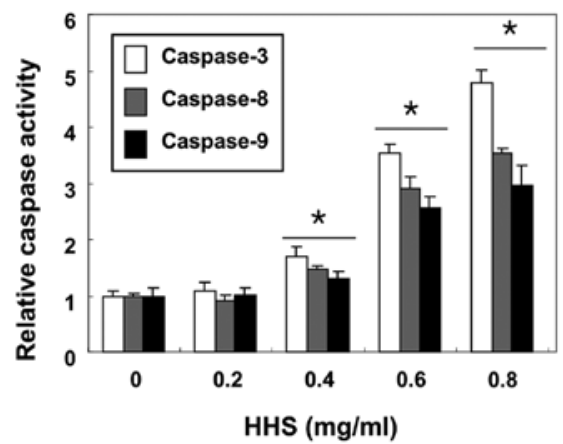

C

Caspase-3

Caspase-8

Caspase-9

PARP

Actin

HHS $(\mathrm{mg} / \mathrm{ml})$

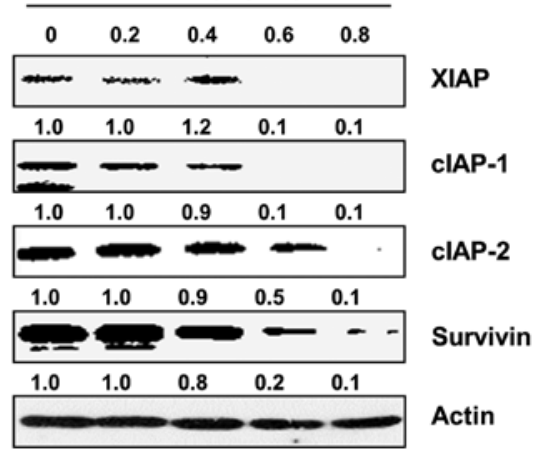

Figure 3. Activation of caspases, degradation of PARP and downregulation of IAP family protein expression by HHS treatment in HCT116 cells. The cells were treated with the indicated concentrations of HHS for $48 \mathrm{~h}$. (A and C) The cells were lysed, and cellular proteins were separated by SDS-polyacrylamide gel electrophoresis and transferred to nitrocellulose membranes. The membranes were probed with the indicated antibodies. Proteins were visualized using an ECL detection system. To confirm equal loading, actin was used as an internal control. The numbers represent the average densitometric analyses as compared with actin in, at a minimum, two or three different experiments. (B) The cells were lysed and aliquots (50 $\mu \mathrm{g}$ of protein) were assayed for caspase- $3,-8$ and -9 activity using DEVD-pNA, IETD-pNA and LEHD-pNA as substrates, respectively. The fluorescent products released were measured ("p $<0.05$ vs. the untreated control).

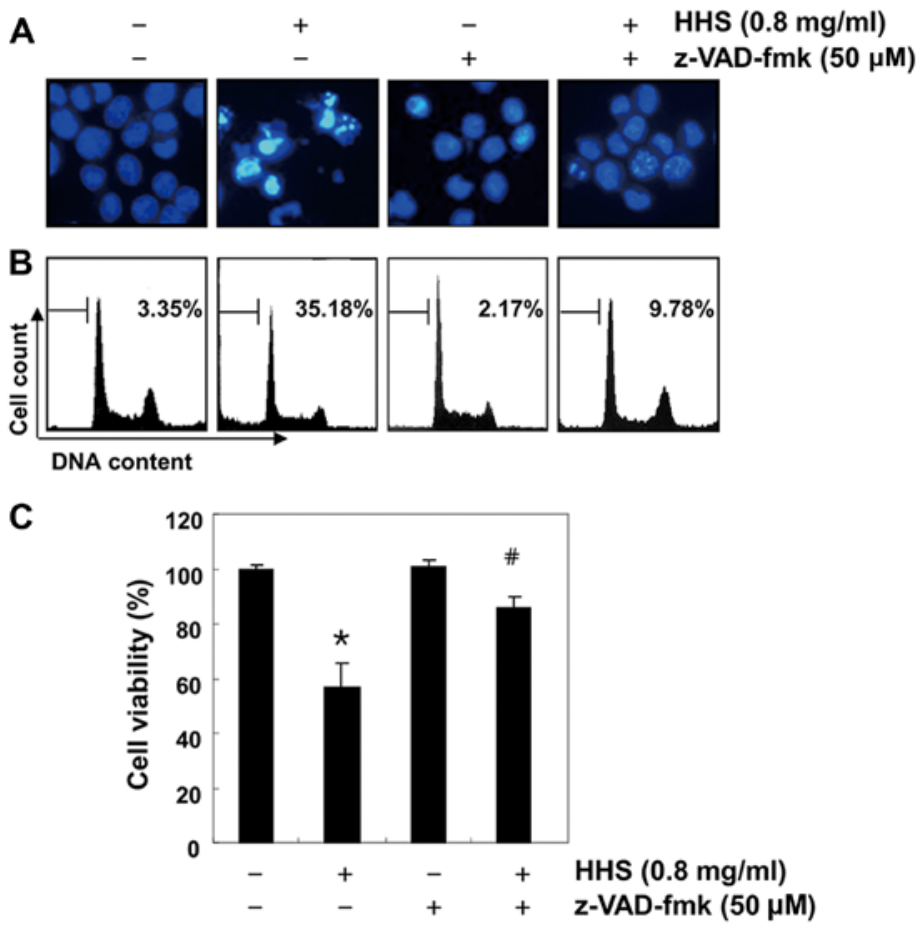

Figure 4. Inhibition of HHS-induced apoptosis by pan-caspase inhibitor in HCT116 cells. The cells were pre-treated for $1 \mathrm{~h}$ with or without $\mathrm{z}$-VAD-fmk $(50 \mu \mathrm{M})$ and then treated with HHS $(0.8 \mathrm{mg} / \mathrm{ml})$ for an additional $48 \mathrm{~h}$. (A) The cells were stained with DAPI and photographed with a fluorescence microscope (original magnification, $\mathrm{x} 400$ ). (B) The percentages of apoptotic cells (sub-G1 cells) were analyzed using flow cytometric analysis. The results are expressed as the mean of the two different experiments. (C) Cell viability was determined by the MTT assay. Each point represents the mean \pm SD of three independent experiments ( ${ }^{*} \mathrm{p}<0.05$ vs. the untreated control; ${ }^{*} \mathrm{p}<0.05$ vs. the HHS-treated cells). 


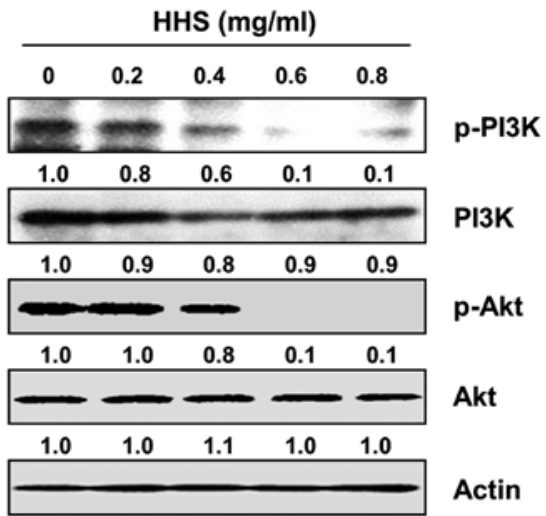

Figure 5. Inactivation of PI3K/Akt signaling by HHS treatment in HCT116 cells. The cells were treated with various concentrations of HHS for $48 \mathrm{~h}$. Equal amounts of cell lysate were resolved by SDS-polyacrylamide gels, transferred to nitrocellulose membranes, and probed with the anti-p-PI3K, anti-p-Akt, anti-PI3K and anti-Akt antibodies. The proteins were visualized using an ECL detection system. Actin was used as an internal control. The numbers represent the average densitometric analyses as compared with actin in, at a minimum, two or three different experiments.

indicating that the HHS-induced activation of caspases was associated with the reduction in the IAP family proteins.

To confirm the involvement of caspase activation in the apoptosis induced by HHS, we conducted a test to determine whether the caspase inhibitor could attenuate the HHS-induced apoptosis and viability reduction. As shown in Fig. 4A and B, pre-treatment with $\mathrm{z}$-VAD-fmk, a pan-caspase inhibitor, resulted in the significant prevention of apoptotic features and the accumulation of hypodiploid cells. The MTT assay also revealed that cells that were co-treated with HHS and z-VAD-fmk had significantly greater cell viability than those that were treated with HHS only (Fig. 4C).

HHS inhibits the activation of PI3K/Akt signaling in the HCT116 cells. The PI3K/Akt pathway has been known to transduce anti-apoptotic signals that confer a survival advantage and resistance of cancer cells against various chemotherapeutic agents $(7,9)$. We therefore sought to determine the involvement of the PI3K/Akt signaling pathway in the HHS-induced apoptosis in HCT116 cells. The constitutive activation of both PI3K and AKT, one of the main downstream effectors of PI3K, was observed in the control cells, whereas HHS treatment significantly suppressed their phosphorylation in a concentration-dependent manner (Fig. 5). The levels of total PI3K and Akt were unaffected by HHS treatment. To determine whether the mechanism in PI3K/Akt signaling is involved in HHS-induced apoptosis, the cells were treated with HHS in the presence or absence of LY294002, a representative PI3K inhibitor. As shown in Fig. 6A and B, the treatment of LY294002 markedly increased the HHS-induced apoptosis, which was demonstrated by nuclear morphological changes and the flow cytometric analysis. In addition, in combination with LY294002, HHS strongly inhibited cell viability (Fig. 6C), indicating that the inactivation of the PI3K/Akt signaling pathway is involved in HHS-induced HCT116 cell apoptosis.

HHS-induced apoptosis is mediated by intracellular ROS generation in the HCT116 cells. Since the increase of intracel-

A
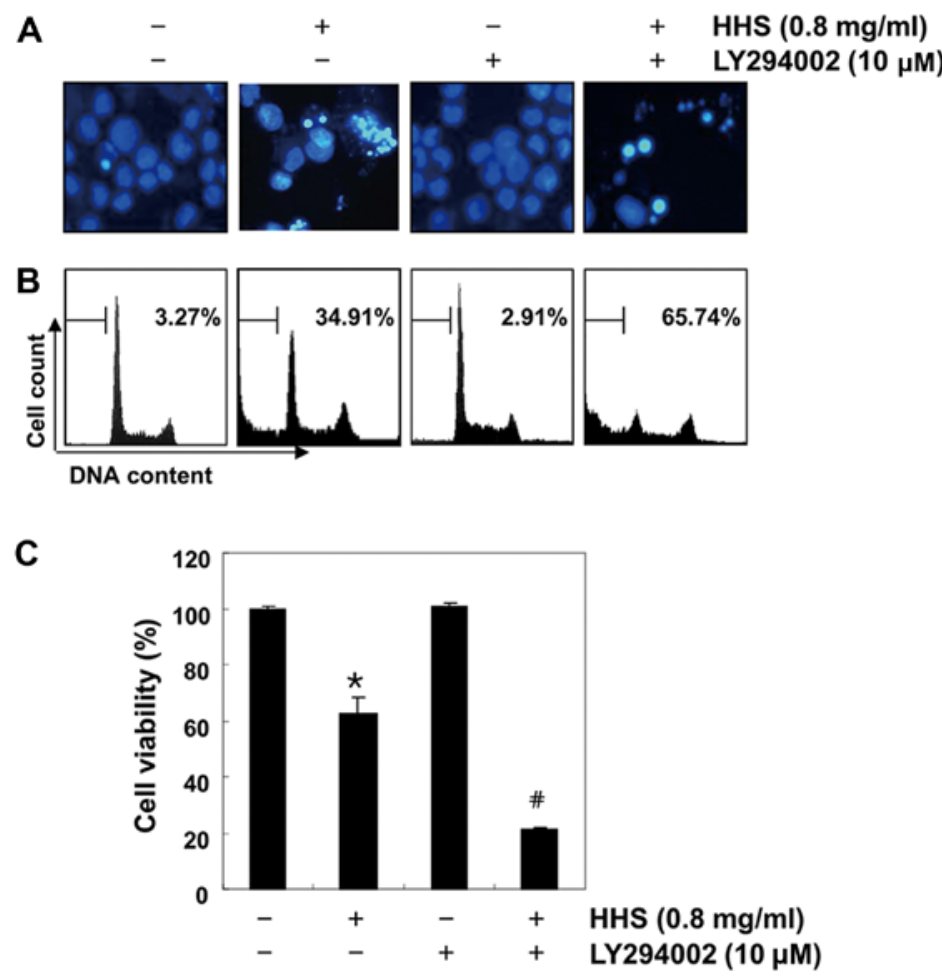

Figure 6. HHS-induced apoptosis is associated with the inactivation of PI3K/Akt signaling in HCT116 cells. The cells were pretreated with PI3K inhibitor (LY294002, $10 \mu \mathrm{M})$ for $1 \mathrm{~h}$ and then treated with HHS $(0.8 \mathrm{mg} / \mathrm{ml})$ for $48 \mathrm{~h}$. (A) After staining with DAPI solution, the nuclei were observed under a fluorescence microscope (original magnification, x400). (B) The percentages of apoptotic cells (sub-G1 cells) were analyzed using flow cytometric analysis. The results are expressed as the mean of the two different experiments. (C) Cell viability was determined by the MTT assay. Each point represents the mean \pm SD of three independent experiments ( $\mathrm{p}<0.05$ vs. the untreated control; ${ }^{*} \mathrm{p}<0.05$ vs. the HHS-treated cells). 

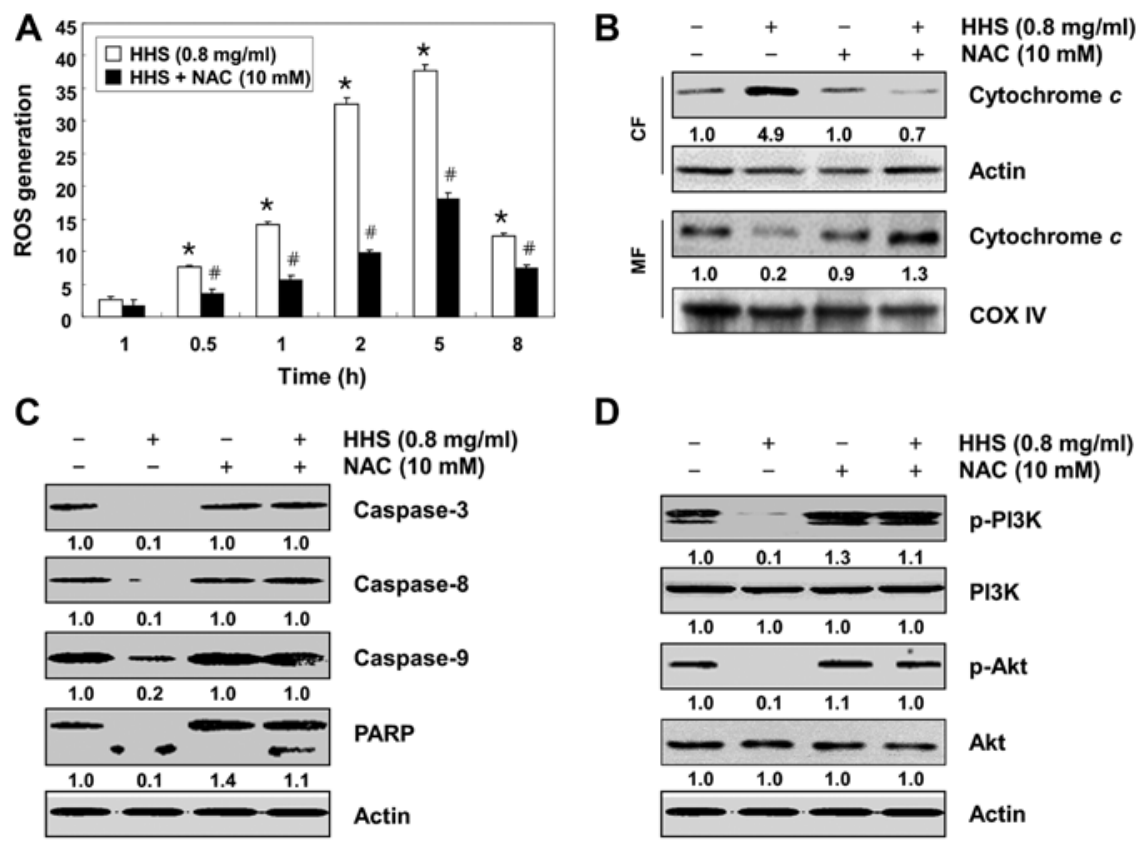

Figure 7. ROS generation by HHS treatment in HCT116 cells. (A) The cells were incubated with HHS (0.8 $\mathrm{mg} / \mathrm{ml})$ for the indicated times or they were pretreated with NAC $(10 \mathrm{mM})$ for $1 \mathrm{~h}$ and further treated with HHS $(0.8 \mathrm{mg} / \mathrm{ml})$ for the indicated times. The cells were then stained with DCFH-DA. ROS generation was measured using a flow cytometry. Each point represents the mean \pm SD of three independent experiments ("p $<0.05$ vs. the untreated control; ${ }^{\#} \mathrm{p}<0.05$ vs. the HHS-treated cells). (B-D) The cells were treated with or without NAC $(10 \mathrm{mM})$ for $1 \mathrm{~h}$ before challenge with HHS $(0.8 \mathrm{mg} / \mathrm{ml})$ for $48 \mathrm{~h}$. They were then harvested, and the indicated proteins were detected by western blot analysis. Actin was used as an internal control. The numbers represent the average densitometric analyses as compared with actin or COX IV in, at a minimum, two or three different experiments.

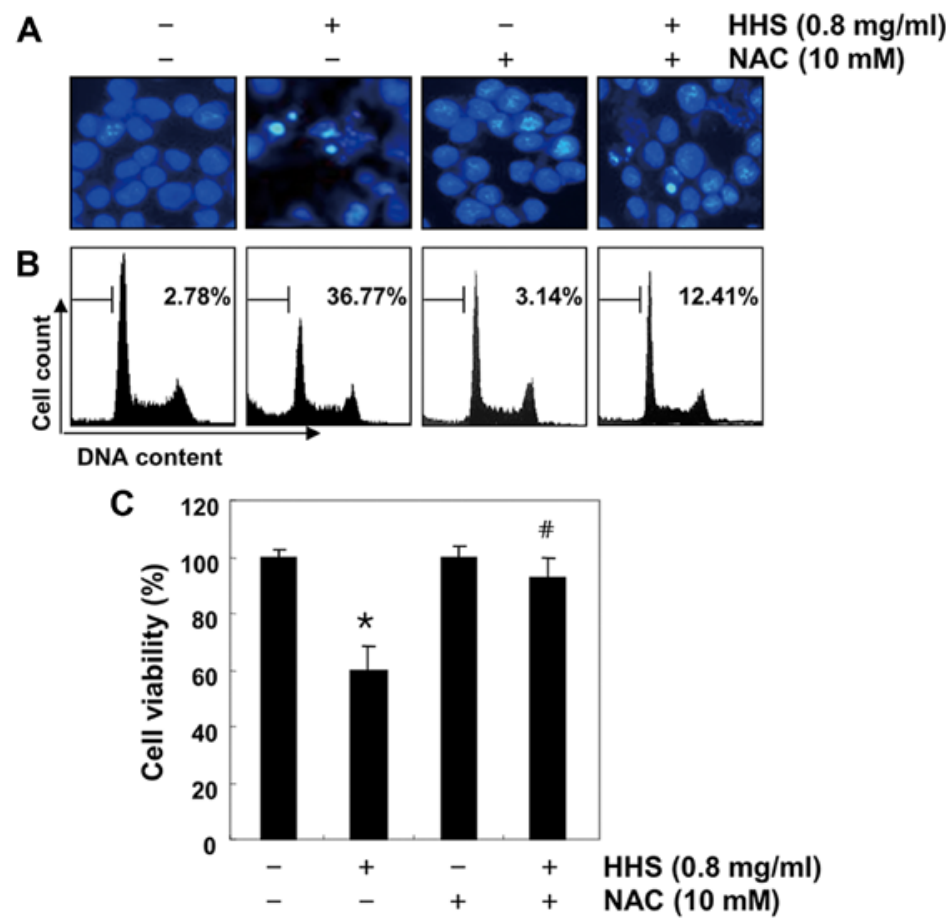

Figure 8. ROS-dependent apoptosis induction by HHS treatment in HCT116 cells. (A) The cells were treated with or without NAC (10 mM) for $1 \mathrm{~h}$ before challenge with HHS $(0.8 \mathrm{mg} / \mathrm{ml})$ for $48 \mathrm{~h}$. (A) They were collected and stained with DAPI to visualize the DNA. The stained nuclei were then observed under a fluorescence microscope (original magnification, $\mathrm{x} 400$ ). (B) To quantify the degree of apoptosis, the cells were evaluated for sub-G1 DNA content using a flow cytometry. The results are expressed as the mean of the two different experiments. (C) Cell viability was determined by the MTT assay. Each point represents the mean \pm SD of three independent experiments ( ${ }^{*} \mathrm{p}<0.05$ vs. the untreated control; ${ }^{*} \mathrm{p}<0.05$ vs. the HHS-treated cells).

lular ROS generation is proposed to be one of the early events in the activation of apoptotic signaling, which alters the cellular redox state and MMP $(8,25)$, we determined whether the generation of ROS is responsible for the apoptosis induced by
HHS in HCT116 cells. The flow cytometric analysis revealed that the generation of ROS was observed at $0.5 \mathrm{~h}$, and the levels continued to increase up to $5 \mathrm{~h}$ in response to the HHS treatment. In contrast, the pretreatment of cells with NAC, a commonly 
used reactive oxygen intermediate scavenger, significantly blocked the HHS-induced increase in ROS generation (Fig. 7A).

In order to show that the generation of ROS is a key step in HHS-induced mitochondrial dysfunction, we compared the levels of cytochrome $\mathrm{c}$ in the cytosolic and mitochondrial fractions. As shown in Fig. 7B, the blocking of ROS generation by the pretreatment of cells with NAC effectively prevented the HHS-induced cytochrome $c$ release into the cytosol, suggesting that the increment in the intracellular ROS level by HHS was linked to mitochondrial dysfunction. NAC pretreatment also markedly prevented the HHS-induced cleavage of caspases (caspase- $8,-9$ and -3 ) and the degradation of PARP (Fig. 7C). Next, we confirmed whether the HCC-induced ROS generation had any functional role in the inactivation of the PI3K/Akt pathway. The results indicated that the pretreatment of HCT116 cells with NAC effectively attenuated the HHS-induced phosphorylation of both PI3K and Akt (Fig. 7D). Furthermore, the apoptotic events in the HHS-treated HCT116 cells were prevented to a considerable extent by the blocking of ROS generation (Fig. 8A and B), which was associated with recovered cell viability, which was revealed by the MTT assay (Fig. 8C), indicating that the HHS-induced inactivation of the PI3K/Akt pathway was mediated by ROS generation.

\section{Discussion}

In the present study, the HHS-induced inhibition of cell proliferation was associated with the induction of apoptotic cell death in HCT116 cells through both the intrinsic and extrinsic pathways. We also demonstrated that exposure of the HCT116 cells to HHS resulted in the induction of apoptosis, which was mediated by ROS generation and the downregulation of the PI3K/Akt signaling cascades.

Since apoptosis plays a crucial role in determining the fate of a cell, apoptosis-inducing agents have been investigated as tools for the management of cancer treatment. In general, apoptosis is initiated by two cellular mechanisms: extrinsic and intrinsic signaling pathways $(5,6)$. In the extrinsic pathway, membrane-bound DRs face the exterior of the plasma membranes to receive stimuli from pro-apoptotic ligands and transmit signals by activating downstream initiators, including caspase- 8 , by the formation of a death-induced signaling complex $(26,27)$. In contrast, one of the key events in the initiation of intrinsic apoptosis is the disintegration of MMP, which induces the release of cytochrome $c$ from the mitochondria to the cytosol and elicits caspase- 9 activation. The activated caspase-8 also directly cleaves Bid to tBid, leading to the activation of the intrinsic pathway through mitochondrial dysfunction, which links the extrinsic pathway to the intrinsic pathway $(5,28)$. Activated caspase- 8 and -9 then promote the cleavage of the effector caspases, including caspase- 3 and -7 , into their active forms, which causes the proteolysis of their substrates, eventually leading to apoptotic cell death $(27,29)$. In the present study, HHS enhanced the expression of FasL, DR4 and DR5, the activation of caspase- 8 and -3 , and the concomitant PARP cleavage with a rise in $\mathrm{tBid}$. We also found that the HHS treatment resulted in the loss of MMP, the release of cytochrome $c$, and the activation of caspase-9. However, pretreatment with the pan-caspase inhibitor, z-VAD-fmk, strongly attenuated HHS-induced cell death and afforded significant protection against HHS-induced growth reduction. Therefore, the results indicate that HHS appears to induce apoptosis primarily through the caspase-dependent, intrinsic and extrinsic apoptotic pathways in HCT116 cells.

In addition, activation of the intrinsic apoptosis pathway is tightly regulated by Bcl-2 family proteins, which are key regulators of mitochondrial membrane function $(26,30)$. In mammalian cells, the anti-apoptotic proteins of the Bcl-2 family, such as Bcl-2 and Bcl-xL, inhibit apoptosis and promote cell survival, which is thought to be involved in the resistance to conventional cancer treatment, including CRC $(31,32)$. However, pro-apoptotic proteins from the same gene family, including Bax and Bad, can critically induce apoptotic cell death. In particular, Bax, in combination with tBid, can induce the release of cytochrome $c$ by creating pore channels in the process of oligomerization in the outer mitochondrial membrane or by opening other channels $(18,19)$. Therefore, apoptosis largely depends on the expression ratio between anti-apoptotic and pro-apoptotic protein levels. Here we found that HHS treatment resulted in a concentration-dependent inhibition of the expression of Bcl-2 protein and a concomitant increase in the expression of Bax. These results suggest that Bax was oligomerized to anchor onto the outer mitochondrial membrane, forming mitochondrial permeability transition pores that disrupted the MMP in the HCT116 cells. Therefore, the disturbance of the $\mathrm{Bcl}-2 / \mathrm{Bax}$ ratio could contribute to the mitochondrial response to the generation of $\mathrm{tBid}$, leading to mitochondrial disturbance and finally releasing cytochrome $c$ and activating caspase-9 to intensify the initial apoptotic response.

Recently, the activation of the PI3K/Akt signaling pathway and its downstream transcription factors has been shown to play a critical role in the control of cellular proliferation $(7,33)$. A growing amount of evidence suggests that PI3K/Akt is activated in most cancer tissues, including CRC, compared to normal tissue. Several studies have shown that the dysregulation of the PI3K/Akt signaling pathway leads to cancer progression $(9,10)$. Moreover, there is much scientific interest in finding molecular pathways and novel compounds that target PI3K/Akt signaling as a new potential therapeutic option for cancer treatment $(10,34)$. Therefore, we further clarified the regulatory role of PI3K/Akt signaling in HHS-mediated HCT116 cell apoptosis. We found that the treatment with HHS had an inhibitory effect on the levels of phosphorylation of AMPK and its downstream target, Akt, in HCT116 cells without the reduction in the steady-state levels of the total PI3K and Akt. To rule out the possibility that the pro-apoptotic effect of HHS was caused by the inactivation of PI3K/Akt after HHS treatment, we pretreated the cells with a synthetic PI3K inhibitor, LY294002. The results indicated that the inhibition of PI3K/Akt signaling in the HCT116 cells effectively protected them from HHS-induced apoptosis and cell viability inhibition. Thus, the results clearly indicate that HHS has anticancer effects with respect to its ability to inhibit the PI3K/Akt signaling pathway, which may be one of the factors leading to apoptosis.

Accumulating evidence indicates that moderate levels of ROS can promote cell proliferation and survival; however, excessive ROS production can induce apoptosis (25). Consistent with this finding, many chemotherapeutic drugs exert their cytotoxic effects through the generation of ROS by causing oxidative 
stress, while many inhibitors of apoptosis show antioxidant activity $(35,36)$. Indeed, the mitochondrial dysfunction induced by excessive ROS generation leads to MMP loss and apoptotic processes (37-39). In the present study, we also found that incubation with HHS significantly increased the ROS level, which was abolished by the pretreatment of cells with the antioxidant NAC. The NAC treatment also rescued cells from HHS-induced apoptosis and growth reduction by blocking the release of cytochrome $c$ and the cleavage of caspases and PARP. Furthermore, it is noteworthy that blocking ROS generation prevented the HHS-induced phosphorylation of PI3K and Akt, demonstrating that HHS stimulated the production of ROS, which subsequently inactivated the PI3K/Akt signaling pathway.

In conclusion, the results indicate that HCT116 cells undergo apoptosis in response to treatment with HHS, which occurs through the activation of the intrinsic and extrinsic pathways, and activation of caspases is responsible for the mediation of HHS-induced apoptosis. In addition, we proposed that the HHS-mediated apoptosis is caused by generation of ROS, which may contribute to the inactivation of PI3K/Akt signaling and the induction of caspase-dependent HCT116 cell apoptosis. Although these findings indicate that ROS play a pivotal role in the regulation of HHS-induced apoptosis in HCT116 cells, further studies are needed to identify the active compounds of HHS. Moreover, elucidation of additional molecular mechanisms and the biological efficacy of HHS may lead to the development of therapeutic approaches for the attenuation of CRC.

\section{Acknowledgements}

The present study was supported by the Basic Science Research Program through the National Research Foundation of Korea (NRF) grant funded by the Korea government (2012046358 and 2015R1A2A2A01004633).

\section{References}

1. Brenner H, Kloor M and Pox CP: Colorectal cancer. Lancet 383: 1490-1502, 2014.

2. Siegel RL, Miller KD and Jemal A: Cancer statistics, 2015. CA Cancer J Clin 65: 5-29, 2015.

3. Hohla F, Winder T, Greil R, Rick FG, Block NL and Schally AV: Targeted therapy in advanced metastatic colorectal cancer: Current concepts and perspectives. World J Gastroenterol 20: 6102-6112, 2014.

4. Fadeel B and Orrenius S: Apoptosis: A basic biological phenomenon with wide-ranging implications in human disease. J Intern Med 258: 479-517, 2005.

5. WalczakH and Krammer PH: The CD95 (APO-1/Fas) and the TRAIL (APO-2L) apoptosis systems. Exp Cell Res 256: 58-66, 2000.

6. Lavrik IN: Systems biology of apoptosis signaling networks. Curr Opin Biotechnol 21: 551-555, 2010.

7. Li ZY, Yang Y, Ming M and Liu B: Mitochondrial ROS generation for regulation of autophagic pathways in cancer. Biochem Biophys Res Commun 414: 5-8, 2011.

8. Nakanishi A, Wada Y, Kitagishi Y and Matsuda S: Link between $\mathrm{PI} 3 \mathrm{~K} / \mathrm{AKT}$ /PTEN pathway and NOX protein in diseases. Aging Dis 5: 203-211, 2014.

9. Pandurangan AK: Potential targets for prevention of colorectal cancer: A focus on PI3K/Akt/mTOR and Wnt pathways. Asian Pac J Cancer Prev 14: 2201-2205, 2013.

10. Danielsen SA, Eide PW, Nesbakken A, Guren T, Leithe E and Lothe RA: Portrait of the PI3K/AKT pathway in colorectal cancer. Biochim Biophys Acta 1855: 104-121, 2015.

11. Yang G, Li X, Li X, Wang L, Li J, Song X, Chen J, Guo Y, Sun X, Wang S, et al: Traditional chinese medicine in cancer care: A review of case series published in the Chinese literature. Evid Based Complement Alternat Med 2012: 751046, 2012.
12. Wang X, Feng Y, Wang N, Cheung F, Tan HY, Zhong S, Li C and Kobayashi S: Chinese medicines induce cell death: The molecular and cellular mechanisms for cancer therapy. Biomed Res Int 2014: 530342, 2014

13. Li J, Lu C, Jiang M, Niu X, Guo H, Li L, Bian Z, Lin N and Lu A: Traditional chinese medicine-based network pharmacology could lead to new multicompound drug discovery. Evid Based Complement Alternat Med 2012: 149762, 2012.

14. Cha WS, Oh JH,Park HJ, Ahn SW, Hong SY and Kim NI: Historical difference between traditional Korean medicine and traditional Chinese medicine. Neurol Res 29 (Suppl 1): S5-S9, 2007.

15. Kwon T, Rho JK, Lee JC, Park YH, Shin HJ, Cho S, Kang YK, Kim BY, Yoon DY and Yu DY: An important role for peroxiredoxin II in survival of A549 lung cancer cells resistant to gefitinib. Exp Mol Med 47: e165, 2015.

16. Kim YS, Li XF, Kang KH, Ryu B and Kim SK: Stigmasterol isolated from marine microalgae Navicula incerta induces apoptosis in human hepatoma HepG2 cells. BMB Rep 47: 433-438, 2014.

17. Song JL, Choi JH, Seo JH, Kil JH and Park KY: Antioxidative effects of fermented sesame sauce against hydrogen peroxideinduced oxidative damage in LLC-PK1 porcine renal tubule cells. Nutr Res Pract 8: 138-145, 2014.

18. Kadenbach B, Arnold S, Lee I and Hüttemann M: The possible role of cytochrome $c$ oxidase in stress-induced apoptosis and degenerative diseases. Biochim Biophys Acta 1655: 400-408, 2004.

19. Scorrano L and Korsmeyer SJ: Mechanisms of cytochrome $c$ release by proapoptotic BCL-2 family members. Biochem Biophys Res Commun 304: 437-444, 2003.

20. Degli Esposti M and Dive C: Mitochondrial membrane permeabilisation by Bax/Bak. Biochem Biophys Res Commun 304: 455-461, 2003.

21. Karbowski M, Norris KL, Cleland MM, Jeong SY and Youle RJ: Role of Bax and Bak in mitochondrial morphogenesis. Nature 443: 658-662, 2006.

22. Duriez PJ and Shah GM: Cleavage of poly(ADP-ribose) polymerase: A sensitive parameter to study cell death. Biochem Cell Biol 75: 337-349, 1997.

23. Danson S, Dean E, Dive C and Ranson M: IAPs as a target for anticancer therapy. Curr Cancer Drug Targets 7: 785-794, 2007.

24. de Graaf AO, de Witte $\mathrm{T}$ and Jansen JH: Inhibitor of apoptosis proteins: New therapeutic targets in hematological cancer? Leukemia 18: 1751-1759, 2004.

25. Fleury C, Mignotte B and Vayssière JL: Mitochondrial reactive oxygen species in cell death signaling. Biochimie 84: 131-141, 2002.

26. Ola MS, Nawaz M and Ahsan H: Role of Bcl-2 family proteins and caspases in the regulation of apoptosis. Mol Cell Biochem 351: 41-58, 2011.

27. Hensley P, Mishra M and Kyprianou N: Targeting caspases in cancer therapeutics. Biol Chem 394: 831-843, 2013.

28. Fulda $S$ and Debatin KM: Extrinsic versus intrinsic apoptosis pathways in anticancer chemotherapy. Oncogene 25: 4798-4811, 2006.

29. Fiandalo MV and Kyprianou N: Caspase control: Protagonists of cancer cell apoptosis. Exp Oncol 34: 165-175, 2012.

30. Tomek M, Akiyama T and Dass CR: Role of Bcl-2 in tumour cell survival and implications for pharmacotherapy. J Pharm Pharmacol 64: 1695-1702, 2012.

31. Koehler BC, Jäger D and Schulze-Bergkamen H: Targeting cell death signaling in colorectal cancer: Current strategies and future perspectives. World J Gastroenterol 20: 1923-1934, 2014.

32. Qiao L and Wong BC: Targeting apoptosis as an approach for gastrointestinal cancer therapy. Drug Resist Updat 12: 55-64, 2009.

33. Marone R, Cmiljanovic V, Giese B and Wymann MP: Targeting phosphoinositide 3-kinase: Moving towards therapy. Biochim Biophys Acta 1784: 159-185, 2008.

34. Moral M and Paramio JM: Akt pathway as a target for therapeutic intervention in HNSCC. Histol Histopathol 23: 1269-1278, 2008.

35. Kardeh S, Ashkani-Esfahani S and Alizadeh AM: Paradoxical action of reactive oxygen species in creation and therapy of cancer. Eur J Pharmacol 735: 150-168, 2014.

36. Matés JM, Segura JA, Alonso FJ and Márquez J: Oxidative stress in apoptosis and cancer: An update. Arch Toxicol 86: 1649-1665, 2012.

37. Jourdain A and Martinou JC: Mitochondrial outer-membrane permeabilization and remodelling in apoptosis. Int $\mathrm{J}$ Biochem Cell Biol 41: 1884-1889, 2009.

38. Seo K, Ki SH and Shin SM: Methylglyoxal induces mitochondrial dysfunction and cell death in liver. Toxicol Res 30: 193-198, 2014.

39. Asakura $\mathrm{T}$ and Ohkawa K: Chemotherapeutic agents that induce mitochondrial apoptosis. Curr Cancer Drug Targets 4: 577-590, 2004. 